\title{
Mariposas Frugívoras Capturadas por Armadilhas Atrativas em Fragmento Urbano
}

\author{
Tatiane Tagliatti Maciel1 ${ }^{\bowtie}$, Bruno Corrêa Barbosa1 ${ }^{1}$, Helba Helena Santos-Prezoto² \& Fábio Prezoto1
}

1. Universidade Federal de Juiz de Fora, e-mail: tatitagliatti@hotmail.com (Autor para correspondência ${ }^{\bowtie}$ ), barbosa.bc@outlook.com, fabio.prezoto@ufjf.edu.br. 2. Centro de Ensino Superior de Juiz de Fora, e-mail: helba.santos@ig.com.br.

\section{EntomoBrasilis 8 (2): 91-95 (2015)}

Resumo. Lepidópteros frugívoros, em geral, apresentam grande importância ecológica, sendo frequentemente utilizados como bioindicadores em estudos de avaliação ambiental. Contudo, as metodologias propostas para a captura de mariposas requerem grande esforço em campo para instalação e monitoramento das armadilhas, além de apresentarem alto custo. Com isso, iscas atrativas têm sido avaliadas para auxiliar os trabalhos de detecção e monitoramento de mariposas. O objetivo do presente estudo foi, portanto, registrar a diversidade da família Noctuidae capturada por armadilhas com atrativos alimentares e avaliar o desempenho desse modelo metodológico para sua utilização em futuros trabalhos com noctuídeos. O estudo foi realizado em área de Fragmento urbano de Floresta Estacional Semidecidual onde foram utilizados três tipos de isca: "Abacaxi com Caldo de Cana", "Banana com Caldo de Cana" e "Banana com Água" e coletados 49 indivíduos da família Noctuidae distribuídos por seis gêneros e onze espécies. A isca "Abacaxi com caldo de cana" foi a que apresentou o maior índice de diversidade, contudo a isca que apresentou maior riqueza e maior dominância foi "Banana com água", já a isca "Banana com caldo de cana" foi a que apresentou o maior índice de equitabilidade. A partir dos resultados, pode-se inferir que o uso de armadilhas com atrativos alimentares, em especial "Banana com água”, pode ser uma alternativa vantajosa em Avaliação Ecológica Rápida ou determinação da presença de alguns grupos como Noctuidae em determinada área, explicitando a necessidade do aprimoramento das armadilhas e testes de iscas para se obter melhor resposta das mariposas à essa metodologia.

Palavras-Chave: Atrativo alimentar; Avaliação Ecológica Rápida; Lepidoptera; Metodologia; Noctuidae.

\section{Frugivorous Moths Captured by Attractive Traps in Urban Fragment}

Abstract. Generally, frugivorous lepidopteran, have great ecological importance and are often used as bioindicator in environmental assessment studies. However, the proposed methodologies for capturing moths require great effort on the field for installation and monitoring of traps, in addition to their high cost. Thereat attractive baits have been evaluated to assist the work of detection and monitoring of moths. The aim of this study was, therefore, to record the diversity of the Noctuidae family captured by traps with food attractions evaluate the performance of this methodology model for its use in future work with cutworms. The study was conducted in urban semideciduous seasonal forest fragment in which three types of bait were used: pineapple with sugarcane juice, banana with sugarcane juice, and banana with water and collected 49 individuals of the Noctuidae family spread over six genera and eleven species. The bait of pineapple with sugarcane juice was the one with the highest diversity index, but the bait that showed greater wealth and greater dominance was banana with water as bait banana with sugarcane juice showed the highest equitability. From the results, it can be inferred that the use of attractive traps can be an advantageous alternative for Rapid Ecological Assessment or determining the presence of some groups as Noctuidae in a given area, explaining the need for the improvement of traps and bait tests to obtain best response of moths to this methodology.

Keywords: Attractive food; Lepidoptera; Methodology; Noctuidae; Rapid Ecological Assessment.

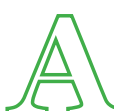

$\mathrm{s}$ constantes modificações na paisagem causadas, sobretudo pelo processo de urbanização, têm levado à destruição, fragmentação e ao isolamento de habitats naturais, com consequente prejuízo para a biodiversidade evidenciando a importância das áreas remanescentes como refúgio para a fauna e flora (Silva et al. 2007). O conhecimento acerca dos organismos presentes em determinados locais como, por exemplo, fragmentos urbanos, é primordial para o desenvolvimento de qualquer estratégia de conservação (SANTos 2003).

Dentre os insetos presentes nestas áreas, destacam-se os Lepidoptera, que apresentam ciclos reprodutivos dinâmicos, respondendo rapidamente às mudanças na vegetação e no clima, sendo facilmente observados e amostrados em qualquer época do ano e por isso valorizados como bons indicadores biológicos (Brown JR 1991; FreITAS et al. 2003). Entre os Lepidópteros estão as mariposas, representadas por 121 famílias (BROWN JR \& FREITAS 1999), cujos hábitos alimentares são bastante variados incluindo néctar, pólen, sangue, secreções e excrementos de animais, seiva, polpa de frutas ou qualquer tipo de substrato capaz de disponibilizar substâncias necessárias para manutenção de suas necessidades metabólicas (SCOBLE 1995).

Além da importância ecológica como bioindicadoras e polinizadoras, em fase de lagarta, mariposas são apontadas como pragas de fruticulturas de pessegueiros, macieiras e pereiras (Queiroz \& CAldas 2012) e citros (Bento \& PARRa 2008), além de apresentarem grande importância médica devido a acidentes com o gênero Lonomia (Pineda et al. 2001; Almeida et al. 2013).

Dessa forma, acredita-se que as mariposas podem ser atraídas por armadilhas com atrativos alimentares, sendo assim os objetivos do presente estudo foram confirmar essa hipótese registrando a diversidade da família Noctuidae capturada por armadilhas com atrativos alimentares e avaliar o desempenho desse modelo de metodologia para a utilização do mesmo em futuros trabalhos com noctuídeos, uma vez que estudos sobre esse grupo e variedade de metodologias para coleta dos mesmos são escassos.

\section{MATERIAL E MÉTODOS}

O trabalho foi realizado no Jardim Botânico da Universidade Federal de Juiz de Fora ( $21^{\circ} 43^{\prime} 28^{\prime \prime} \mathrm{S} ; 43^{\circ} 16^{\prime} 47^{\prime \prime}$ O), fragmento 
de Floresta Estacional Semidecidual Montana (Veloso et al. 1991), localizado no perímetro urbano de Juiz de Fora, sudeste do Estado de Minas Gerais, a $750 \mathrm{~m}$ acima do nível do mar que apresenta clima subtropical quente com inverno seco e verão chuvoso (Cwa), segundo a classificação de Köppen-Geiger (SÁ JúNIOR 2009). A área, de 84 ha de extensão, foi recentemente classificada por SANTiago et al. (2014) como complexo de expressiva riqueza, diversidade e heterogeneidade florística de vegetação arbórea, com espécies ameaçadas de extinção e com predominância de plantas pioneiras, além da presença considerável de espécies exóticas. O período do estudo foi entre julho de 2013 e fevereiro de 2014, totalizando seis coletas, sendo três no período seco (julho, agosto e setembro) e três no período chuvoso (Outubro, Janeiro e Fevereiro).

Um total de 54 armadilhas atrativas do tipo Van Someren-Rydon (Figura 1) (BATRA 2006), com modificações de alguns materiais como os da base, onde a placa de metal foi substituída por um recipiente de plástico, foram instaladas a uma altura aproximada de 1,5 m do solo e divididas em três conjuntos. Cada conjunto foi composto por três armadilhas atrativas, contendo $200 \mathrm{~mL}$ de isca (uma de cada tipo), distantes $25 \mathrm{~m}$ entre si. Os conjuntos foram separados por uma distância de $225 \mathrm{~m}$ e permaneceram em campo por $48 \mathrm{~h}$ por coleta, perfazendo um total de $288 \mathrm{~h}$ de esforço amostral. As iscas utilizadas foram as mesmas propostas para lepidópteros frugívoros, "Abacaxi com Caldo de Cana" (Uehara-Prado 2004), "Banana com Caldo de Cana" (Silva et al. 2012) e "Banana com Água" (Silva et al. 2010), preparadas a uma proporção de três partes de fruta pra uma de líquido (3:1) e fermentadas previamente por 48 horas (BATRA 2006).

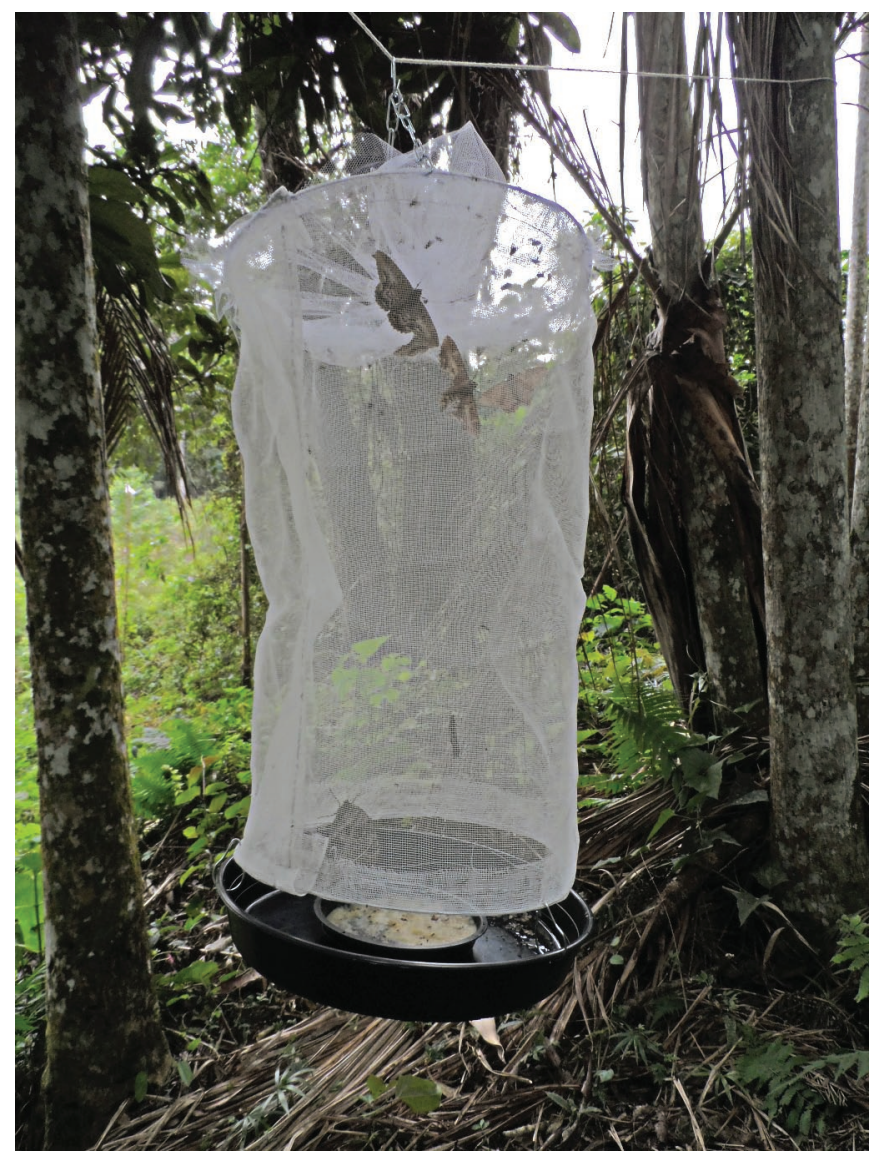

Figura 1. Armadilha atrativa do tipo Van Someren-Rydon em campo.

Os noctuídeos de pequeno porte capturados foram mortos por compressão do tórax, já os indivíduos maiores, foram mortos injeção de uma pequena quantidade de etanol no tórax, seguindo-se a metodologia proposta por DUARTE et al. (2012). Todos os espécimes foram armazenados em envelopes entomológicos para posterior identificação.
As identificações foram realizadas através de observação da presença da abertura timpânica entre o metatórax e o abdome, caractere exclusivo de Noctuidae e de consulta na coleção entomológica da Reserva Técnica do Museu de História Natural da Academia de Comércio e do Centro de Ensino Superior de Juiz de Fora e revistas pelo Prof. Maurício Moraes Zenker da Universidade Federal do Paraná, especialista em taxonomia de Arctiinae (Noctuidae). Os exemplares, cuja identificação não pôde ser prontamente realizada, foram preparados em alfinetes entomológicos e incluídos na listagem ao nível de gênero. Alguns indivíduos foram montados em via seca para compor a caixa testemunha e foram depositados no Laboratório de Ecologia Comportamental e Bioacústica (LABEC) da Universidade Federal de Juiz de Fora.

A diversidade foi calculada para cada isca utilizando-se o Índice de Shannon-Wiener ( $\left.\mathrm{H}^{\prime}\right)$, para o índice de dominância das iscas foi utilizado o Teste de Berger-Parker e o valor de equitabilidade foi feito pelo Teste de Simpson (ED). Todos os testes foram realizados através do software freeware DivEs 3.0 (RoDRIGUES 2014). Já para análise de Variância de Abundância e Riqueza, foi usado o teste Kruskal-Wallis (P), pelo software freeware BioEstat $® 5.3$.

\section{RESULTADOS E DISCUSSÃO}

Foram coletados 49 indivíduos da família Noctuidae, distribuídos por seis gêneros e onze espécies (Tabela 1), sendo a espécie Letis buteo Genée a mais abundante representando $41 \%$ do total de indivíduos coletados. A isca "Banana com água" (BA) capturou a maior parte dos indivíduos coletados $(53 \% ; \mathrm{N}=26)$, seguido de "Abacaxi com caldo de cana" (AC) $(35 \% ; \mathrm{N}=17)$ e "Banana com caldo de cana" (BC) (12\%; $\mathrm{N}=6)$. Entretanto, quando aplicado o teste estatístico Kruskal-Wallis, as iscas não apresentaram diferença significativa (BAxAC: $\mathrm{H}=0,5247$ / $\mathrm{p}=0,4688$; $\mathrm{BAxBC}$ : $\mathrm{H}=2,6097 / \mathrm{p}=0,1062 ; \mathrm{ACxBC}: \mathrm{H}=1,2788 / \mathrm{p}=0,2581)$. Essa mesma ordem se manteve em relação à riqueza, onde "Banana com água" também capturou a maior parte dos indivíduos coletados (73\%; N=8), seguido por "Abacaxi com caldo de cana" ( $54 \% ; \mathrm{N}=6)$ e "Banana com cana" (45\%; N=5). Contudo, também não houve diferença significativa quando aplicado o teste estatístico Kruskal-Wallis (BAxAC: $\mathrm{H}=0,9635$ / $\mathrm{p}=0,3263$; BAxBC: $\mathrm{H}=2,1444$ / $\mathrm{p}=0,1431$; ACxBC: $\mathrm{H}=0,6918 / \mathrm{p}=0,4055)$.

Através do índice de diversidade de Shannon-Wiener calculado para as iscas, "Abacaxi com caldo de cana" foi a que apresentou o maior índice, contudo a isca que apresentou maior riqueza e maior dominância, indicadas pelo Teste de Simpson, foi "Banana com água”, dominância superior justificada pelo grande número de L. buteo coletada por ela. Já a isca "Banana com caldo de cana" foi a que apresentou o maior índice de equitabilidade, calculado pelo Teste de Simpson (Tabela 1).

Quanto à influência de fatores abióticos como temperatura e umidade relativa do ar, os meses secos e frios (Maio a Setembro) apresentaram uma maior taxa de captura dessas mariposas $(71 \%$ do total) (Figura 2), o que pode ser justificado pelo fato de ser uma época onde os recursos estão mais escassos e as iscas podem ter se tornado uma importante fonte alternativa de alimento e ainda pelo mês de Outubro não ter havido captura por nenhuma das armadilhas. Essa não captura pode ser justificada pela média de velocidade do vento ter sido de $2,4 \mathrm{~m} / \mathrm{s}$ (metros por segundo) na semana da coleta, tendo em vista que velocidades acima de 1 $\mathrm{m} / \mathrm{s}$ já diminuem significativamente a atividade de forrageio dos insetos (CASTRO et al. 2011).

O número de mariposas capturadas no presente estudo foi inferior a de outros trabalhos como LANDOLT et al. (2011), que encontraram 69 espécies da família Noctuidae em trabalho avaliando o desempenho de atrativos químicos a base de solução de fermentado de melaço e de odores de flores em Mata Ciliar. Essa diferença pode ser justificada pelos seguintes fatores: baixa 
Tabela 1 - Lista de espécies e frequência de captura em relação às iscas utilizadas, seguidos de seus índices de diversidade, dominância e equitabilidade no Jardim Botânico da Universidade Federal de Juiz de Fora. Legenda: BA = Banana e Água, AC = Abacaxi e Cana de açúcar e BC = Banana e Cana de açúcar.

\begin{tabular}{|c|c|c|c|}
\hline \multirow{2}{*}{$\begin{array}{c}\text { Família/Espécie } \\
\text { Noctuidae }\end{array}$} & \multicolumn{3}{|c|}{ Iscas Atrativas } \\
\hline & BA & AC & BC \\
\hline Ascalapha odorata (Linnaeus) & 1 & 3 & 1 \\
\hline Letis magna (Gmelin) & 2 & o & 0 \\
\hline Letis sp1 & 4 & 2 & 2 \\
\hline Letis sp2 & o & 2 & o \\
\hline Letis $\mathrm{sp} 3$ & o & o & 1 \\
\hline Letis buteo Genée & 13 & 6 & 1 \\
\hline Cyclopis caecutiens (Hübner) & 2 & 2 & o \\
\hline Ophisma tropicalis Guenée & o & o & 1 \\
\hline Ramphia albizona (Latreille) & 1 & o & o \\
\hline Zale peruncta (Guenée) & 1 & o & 0 \\
\hline Zale sp1 & 2 & 2 & o \\
\hline Índices & BA & AC & BC \\
\hline No Total de espécies & 8 & 6 & 5 \\
\hline № Total de indivíduos & 28 & 17 & 6 \\
\hline Shannon-Wiener & 0,696 & 0,73 & 0,678 \\
\hline Berger-Parker & 0,5 & 0,353 & 0,333 \\
\hline Simpson & 0,805 & 0,947 & 0,972 \\
\hline
\end{tabular}

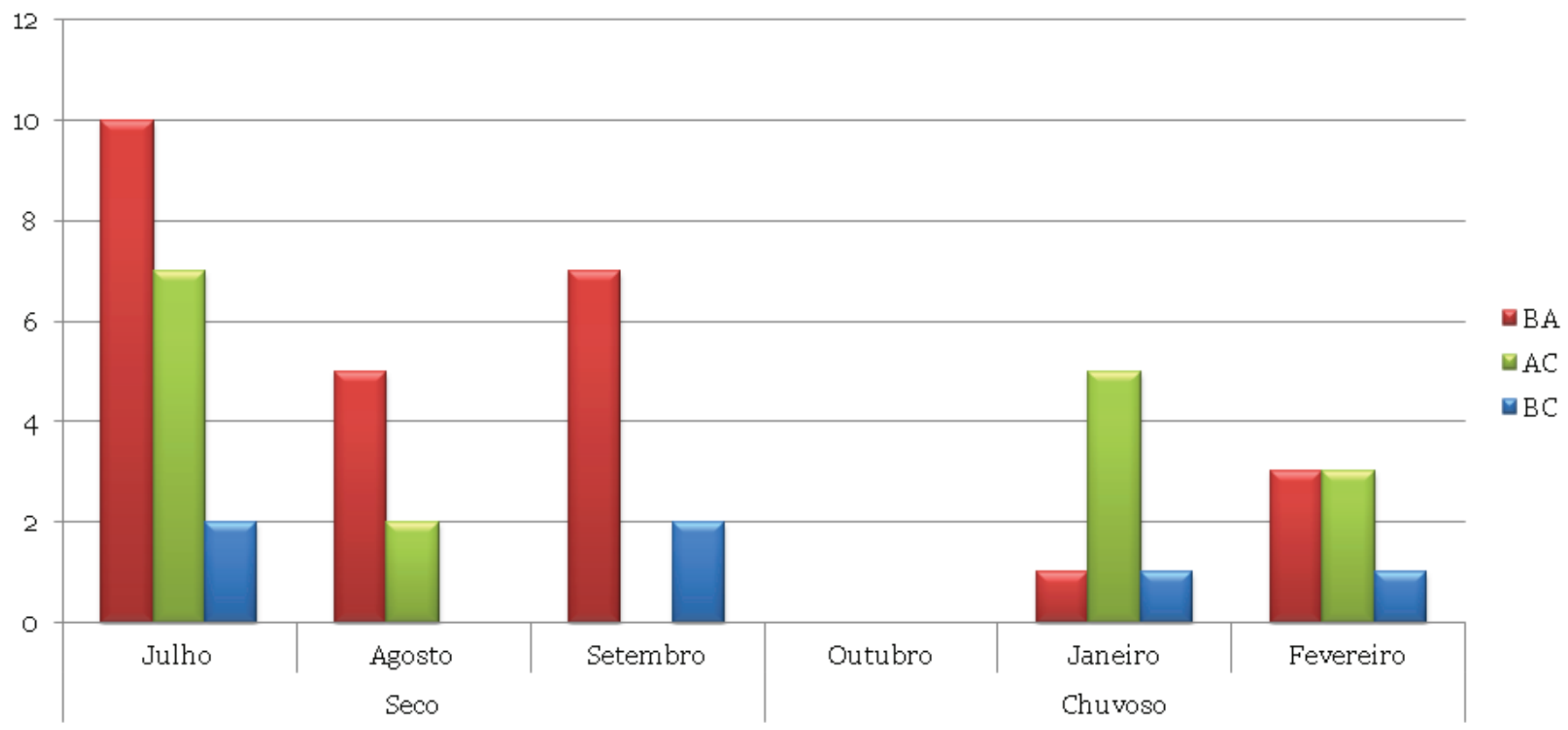

Figura 2. Mariposas capturadas por armadilhas atrativas por cada isca nos períodos Seco e Chuvoso. Legenda: $\mathrm{BA}=\mathrm{Banana}$ e Água, $\mathrm{AC}=\mathrm{Abacaxi}$ e Cana de açúcar e $\mathrm{BC}=$ Banana e Cana de açúcar.

densidade populacional nos locais de instalação das armadilhas e/ ou baixa taxa de resposta das mariposas pelas iscas ou até mesmo pela metodologia empregada. Deve ser considerada ainda a não disponibilidade de cana de açúcar em ambiente natural, inferindo assim que muitas mariposas procuram alimento em outras fontes de açúcar fermentado como sucos, frutas e honeydews (ZENKER et al. 2012), fato confirmado pelo melhor desempenho da única isca sem caldo de cana, "Banana com água" e ainda pelo mesmo trabalho de LANDOLT et al. (2011), que concluíram maior desempenho do atrativo a base de odores de flores.
Em seu estudo em videiras na Serra Gaúcha, Zenker (2012) avaliou duas metodologias de captura de mariposas: metodologia generalista (Armadilha Luminosa) e especialista (Armadilha Mcphail) e encontrou 187 e quatro espécies de Noctuidae, respectivamente, sendo que uma delas foi capturada exclusivamente pela armadilha Mcphail. Apesar da diferença amostrada, por se tratar de uma metodologia em que é necessário grande esforço em campo para instalação e monitoramento das armadilhas e, sobretudo de alto custo, levando em conta ainda que seria inviável para levantamentos em parques por conta da iluminação artificial (FRANK 1988), Armadilhas Luminosas devem 
- ser priorizadas para estudos mais complexos, como amostragens de populações de espécies praga, estudos de dinâmica, ecologia de populações, migração, aspectos comportamentais de voo e relacionamento com as plantas hospedeiras (CHEY et al. 1997; USHER \& KEILLER 1998).

Já para estudos de avaliação ecológica rápida ou para verificação da presença de alguns grupos como Noctuidae em determinada área, para estudos mais específicos, as armadilhas com atrativos alimentares são mais vantajosas e economicamente mais viáveis, pois permanecem em campo $24 \mathrm{~h}$ capturando lepidópteras diurnas e noturnas, possibilitando ainda o estudo de vários grupos de insetos ao mesmo tempo, apresentando melhor desempenho em épocas frias e secas, como mostrado no presente estudo.

Outro campo em que a metodologia apresentada pode ser utilizada, é no diagnóstico de presença ou ausência de pragas agrícolas como Grapholita molesta Busck e Ecdytolopha aurantiana Lima as quais são atualmente monitoradas pelo uso de semioquímicos, como relatam QueIroz \& CALDAS (2012) em seu trabalho com G. molesta e BENTO et al. (2008) com E. aurantiana. Contudo, o desenvolvimento de semioquímicos específicos para cada espécie demanda tempo e dinheiro, motivo pelo qual ainda é pouco disseminado em fruticulturas no Brasil. Assim, o uso de armadilhas com atrativos alimentares, pode ser uma alternativa viável para o monitoramento de pragas para os fruticultores.

Portanto, tendo em vista a carência de estudos com mariposas no Brasil, sobretudo na região Sudeste, e a partir dos resultados obtidos pelo presente estudo, pôde-se inferir que o uso de armadilhas com atrativos alimentares, em especial "Banana com água”, pode ser uma alternativa vantajosa em Avaliação Ecológica Rápida ou determinação da presença de alguns grupos como Noctuidae em determinada área, explicitando a necessidade do aprimoramento das armadilhas e testes de iscas para se obter melhor resposta das mariposas à essa metodologia. Vale ressaltar que, baseado nos resultados de outros trabalhos de diversidade, para levantamentos de longa duração, o mais indicado seria o consórcio de várias metodologias.

\section{REFERÊNCIAS}

Almeida, M., M.M. Castro, N. Bernardo, L.M. Lorini \& A.F.S.F. Rodrigues, 2013. Lonomia obliqua Walker, 1855(Lepidoptera, Saturniidae): First report in the Zona da Mata Mineira region, Southeast Brazil. Check List, 9: 1521-1523.

Batra, P., 2006. Tropical ecology, assessment, and monitoring (team) initiative: Butterfly Monitoring Protocol, $17 \mathrm{p}$.

Bento, J.M.S. \& J.R.P. Parra, 2008. Bicho-Furão-dos-Citros e seu manejo utilizando feromônio sexual, p. 155-168. In: Yamamoto, P.T. (Org.). Manejo Integrado de Pragas dos Citros, Araraquara: Fundecitrus, $336 \mathrm{p}$

Brown Jr, K.S., 1991. Conservation of Neotropical environments: insects as indicators, p. 349-404. In: Collins, N.M. \& A Thomas. The conservation of insects and their habitats. Academic Press, London, 450 p.

Brown JR., K.S. \& A.V.L. Freitas, 1999. Lepidoptera, p. 227243. In: Brandão, C.R.F. \& E.M. Cancello. Biodiversidade do estado de São Paulo, Brasil: síntese do conhecimento ao final do século XX, invertebrados terrestres. São Paulo: FAPESP, $279 \mathrm{p}$.

Castro, M.M., D.L. Guimaraes \& F. Prezoto, 2011. Influence of Environmental Factors on the Foraging Activity of Mischocyttarus cassununga (Hymenoptera, Vespidae). Sociobiology, 58: 133-141.

Chey, V.K., L.O. Holloway \& M.R. Speight, 1997. Diversity of moths in forest plantations and natural forests in Sabah. Bulletin of Entomological Research, Canterbury, 87: 371385 .

Duarte, M., G. Marconato, A. Specht \& M.M. Casagrande, 2012. Lepidoptera, p. 625-682. In: Rafael, J.A., G.A.R. Melo, C.J.B. Carvalho, S.A. Casari \& R. Constantino. (Eds.). Insetos do
Brasil: Diversidade e Taxonomia. Ribeirão Preto: Holos, 810 $\mathrm{p}$.

Frank, K.S., 1988. Impact of outdoor lighting on moths: an assessment. Journal of the Lepidopterists' Society, 42: 6393.

Freitas, A.V.L., R.B. Francini \& K.S. Brown Jr., 2003. Insetos como indicadores ambientais, p. 125-151. In: Cullen, L. Jr., R. Rudran \& C. Valladares-Pádua. Métodos de estudo em Biologia da Conservação e Manejo da Vida Silvestre. Fundação Boticário e Editora da UFPR, Curitiba, 652 p.

Landolt, P.J., T. Adams, R.S. Zack \& L. Crabo, 2011. A diversity of moths (Lepidoptera) trapped with two feeding attractants. Annals of the Entomological Society of America, 104: 498506.

Pineda D., A. Amarillo, J. Becerra \& G. Montenegro, 2001. Sindrome hemorrágico por contacto con orugas del género Lonomia (Saturniidae) en Casanare, Colombia: informe de dos casos. Biomédica, 21: 328-32.

Queiroz; P.R.M. \& B.M.S., Caldas, 2012. Organofosforados e semioquímicos no controle da Grapholita molesta. Ensaios e Ciência: Ciências Biológicas, Agrárias e da Saúde, 16: 115133.

Rodrigues, W.C., 2014. DivEs - Diversidade de Espécies v3.o Guia do Usuário. Entomologistas do Brasil. 3op. Disponível em: http://dives.ebras.bio.br

Sá Júnior, A., 2009. Aplicação da classificação de Köppen para o zoneamento climático do estado de Minas Gerais. Dissertação (Ph.D. em Engenharia Agrícola) - Universidade Federal de Lavras, $101 \mathrm{p}$.

Santiago, D.S., C.R. Fonseca \& F.A. Carvalho, 2014. Fitossociologia da regeneração natural de um fragmento urbano de Floresta Estacional Semidecidual (Juiz de Fora, MG). Revista Brasileira de Ciências Agrárias, 9: 117-123.

Santos, A.J., 2003. Estimativas de riqueza em espécies, p. 1942. In: Cullen Junior, L., R. Rudran \& C. Valladares-Padua. Métodos de estudos em biologia e manejo da vida silvestre. Editora da UFPR, Curitiba, $652 \mathrm{p}$.

Scoble, M.J., 1995. The Lepidoptera form, function and diversity. New York, Oxford University, 404 p.

Silva, A.R.M., M.P.M. Guimarães, R.F. Vitalino, A.S. Bagni, Y.E. Martins, A.M. Cordeiro \& E.G. Oliveira, 2010. Borboletas frugívoras do Parque Estadual do Rio Doce/MG. MGBiota, 3: 5-21.

Silva, A.R.M., C.O.D. Castro, P.O. Mafia, M.O.C. Mendonça, T.C.C. Alves \& M.D.V. Beirão, 2012. Borboletas frugívoras (Lepidoptera: Nymphalidae) de uma área urbana (Área de Proteção Especial Manancial Cercadinho) em Belo Horizonte, Minas Gerais, Brasil. Biota Neotropica, 3: 292 -297.

Silva, A.R.M., G.G. Landa \& R.F. Vitalino, 2007. Borboletas (Lepidoptera) de um fragmento de mata urbano em Minas Gerais, Brasil. Lundiana, 8: 137-142.

Uehara-Prado, M., A.V.L. Freitas, R.B. Francini \& K. Brown Jr., 2004. Guia das borboletas frugívoras da reserva estadual do morro grande e região de Caucaia do Alto, Cotia (São Paulo). Biota Neotropica, 4: 1-9.

Usher, M.S. \& S.W.J. Keiller, 1998. The macrolepidoptera of farm woodlands: determinants of diversity and community structure. Biodiversity and Conservation, 7: 725-748.

Veloso, H.P., A.L. Rangel Filho \& J.C.A. Lima, 1991. Classificação da vegetação brasileira adaptada a um sistema universal. Rio de Janeiro: IBGE, 124p.

Zenker, M.M., 2012. Mariposas em parreirais na região da Serra Gaúcha e informações sobre espécies com potencial para perfurar frutos. EMBRAPA (Circular Técnica Embrapa Uva e Vinho, 89).

Zenker, M.M., M. Botton, J.A. Teston \& A. Specht, 2012. Noctuidae moths occurring in grape orchards in Serra Gaúcha, Brazil and their relation to fruit-piercing. Revista Brasileira de Entomologia, 54: 288-297. 
Recebido em: 10/10/2014

Aceito em: 04/02/2015

Como citar este artigo:

Maciel, T.T., B. Corrêa Barbosa, H.H. Santos-Prezoto \& F. Prezoto, 2015. Mariposas Frugívoras Capturadas por Armadilhas Atrativas em Fragmento

Urbano. EntomoBrasilis, 8 (2): 91-95.

Acessível em: doi:10.12741/ebrasilis.v8i2.480
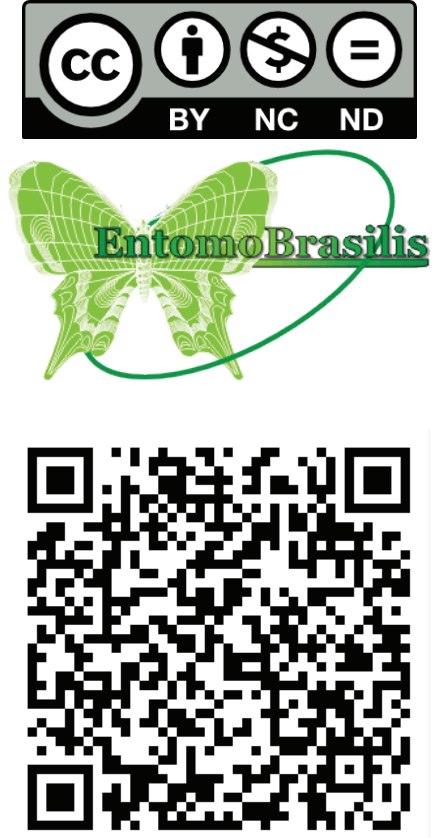